\title{
Rifampicin Bioavailability in Fixed-Dose Combinations for Tuberculosis Treatment: Evidence and Policy Actions
}

\author{
Pankaj Sadaphal', Krishnapada Chakraborty', Hala Jassim-AlMossawi', Yogan Pillay², Giorgio Roscigno ${ }^{3}$, Anil \\ $\mathrm{Kaul}^{4}{ }^{4}$ Neeraj Kak ${ }^{1 *}$, Refiloe Matji' , Lindiwe Mvusi ${ }^{2}$, Anthony DeStefano' \\ 'University Research Co. LLC, Maryland, USA \\ ${ }^{2}$ Department of Health South Africa, Pretoria, South Africa \\ ${ }^{3}$ NEXT2People Foundation, Geneva, Switzerland \\ ${ }^{4}$ Center for Health Sciences, Oklahoma State University, Tulsa, OK
}

\section{Article Info}

\section{Article Notes}

Received: June 21, 2018

Accepted: August 16, 2019

\section{${ }^{*}$ Correspondence:}

Dr. Neeraj Kak, University Research Co., LLC (URC), Chevy Chase, MD, USA; Email: nkak@urc-chs.com.

(C) 2019 Kak N. This article is distributed under the terms of the Creative Commons Attribution 4.0 International License.

\section{Keywords:}

Rifampicin

Combination Drug Therapy

Pharmacokinetics

Bioavailability

Fixed Dose Combinations

Tuberculosis

\section{Abstract}

Purpose: The pharmacokinetics (PK) of anti-tuberculosis drugs, including their bioavailability (BA), significantly impacts the efficacy and effectiveness of tuberculosis (TB) treatment regimens. Rifampicin, one of the most important drugs in the treatment of drug-sensitive tuberculosis, has been used increasingly in fixed-dose combinations (FDCs). This paper reviews and analyzes available data on BA and PK of rifampicin with a focus on FDCs, from published studies and reports.

Methods: Using PubMed as the primary database, Cochrane and other relevant databases, a systematic review of literature was conducted to identify studies on the bioavailability and efficacy of rifampicin in FDCs versus single drug formulations. A number of keywords including "bioavailability", "rifampicin", "fixed dose combinations", and "pharmacokinetics" were used in various combinations. The search covered the period 1980 to 2016 . Priority was given to articles on rifampicin bioavailability in fixed dose combinations used in the program setting, and human studies that used the World Health Organization (WHO) approved BA/PK protocol and sample size ( $\geq 22$ patients).

Findings: More than 450 original peer-reviewed articles, reviews and reports, were assessed for this analysis. Eleven papers, which included data from high-TB-burden countries (South Africa, India, and China), raised significant concerns about rifampicin bioavailability within FDCs; the authors of the studies discussed multiple factors associated with low bioavailability, including drug formulation and quality, storage environment, patient factors, and concomitant diseases.

Implications: Recent studies and reviews point to the problem of low bioavailability of rifampicin in fixed dose combinations. However, in the field, it remains a hidden or unrecognized factor leading to poor treatment outcomes. It is difficult to study the issue thoroughly unless there is awareness among TB program personnel of its existence, and adequate laboratory and research support is available to national tuberculosis programs (NTPs). In stemming the tide of tuberculosis multi-drug resistance (MDR) and extensive drug resistance (XDR), it is paramount to ensure that rifampicin bioavailability is adequate in FDCs, and to detect and address any deviation from recommended target ranges. There is a need for strategies to minimize the undesirable clinical effects of reduced rifampicin bioavailability in FDCs, and for effective utilization of quality-assured drugs within NTPs programs; these can help NTPs support effective case management in line with the international TB care standards, while taking into consideration the factors affecting drug absorption and therapeutic concentration.

\section{Introduction}

The emergence of drug-resistant tuberculosis (DR-TB) is a major 
public health concern across the globe and particularly in tuberculosis (TB) high-burden countries. There are alarming reports of increasing drug resistance from various parts of the world which potentially threaten to disrupt the gains achieved in tuberculosis (TB) control over the last decade $^{1}$ and global progress towards achieving the targets of the End TB strategy. In 2017, there were 558,000 new TB cases with resistance to rifampicin, the most effective first-line drug, of which $82 \%$ had multidrug-resistant TB (MDR-TB) ${ }^{2}$. Rifampicin resistant TB (RR-TB) is defined as resistance to rifampicin detected using genotypic or phenotypic methods with or without resistance to other first-line anti-TB drugs. MDR-TB is essentially a man-made phenomenon and arises due to inadequate treatment of drug-sensitive TB (DS-TB) ${ }^{3}$. The prevalence of MDR-TB mirrors the functional state and efficacy of tuberculosis control programs in the country.

The pharmacokinetics (PK) of anti-tuberculosis (antiTB) drugs, including their bioavailability (BA), have a significant impact on the effectiveness of TB treatment regimens, which are complex and consist of at least four initial drugs. Rifampicin (Rifampin, USP) remains one of the most important drugs in the treatment of drug-sensitive tuberculosis and has been used increasingly in fixed-dose combinations (FDCs) ${ }^{4}$. The World Health Organization (WHO) and International Union Against Tuberculosis and Lung Disease (IUATLD) ${ }^{5}$ recommend FDCs because they improve patient adherence, simplify prescribing and management of drug supplies, and reduce prescription errors. The objective of this paper is to review and analyze the available data on BA and PK of rifampicin from published studies and reports, with a focus on FDCs. Based on these analyses, possible strategies are discussed for minimizing undesirable clinical effects of reduced bioavailability of rifampicin in FDCs and for effective utilization of qualityassured drugs within National Tuberculosis Programs (NTP).

\section{Methods}

A literature search was conducted using PubMed (https://www.ncbi.nlm.nih.gov/pubmed/) as the primary database, for articles related to the bioavailability of rifampicin and FDCs. The Cochrane Library, Cochrane Database of Systematic Reviews, and related reports from WHO/IUATLD were also accessed. A number of keywords including "bioavailability", "rifampicin", "fixed dose combinations", and "pharmacokinetics" were used in various combinations, either as a title word or as a Medical Subject Heading (MeSH). The search covered the period from 1980 to 2016. A reference database was created in Reference Manager and imported into Mendeley software. After implementation of multiple search strategies, duplicate references / citations and those that were not relevant to the present review were identified and removed from the database manually. The relevant full-text articles from those shortlisted (retained) in the database were accessed and reviewed using library sources and online repositories e.g. Elsevier, Wiley, Athens etc. In the review of full-text articles and original papers, priority was given to articles on rifampicin bioavailability in fixed dose combinations used in the program setting. Human studies dealing with BA/PK of rifampicin that used the WHO approved BA/PK protocol for determination of bioavailability of rifampicin in FDCs and met or exceeded the recommended sample size ( $\geq 22$ patients), were selected preferentially ${ }^{6-7}$, while a limited number of studies with fewer subjects that had direct relevance to the subject of interest were also assessed. Laboratory studies, regardless of sample size, were given the next priority and reviewed for BA / PK / bioequivalence (BE) characteristics of rifampicin (and its dissolution) in FDCs.

For each of the articles / studies reviewed, the outcome measures of interest were classified into one of two categories: (a) studies that provided information on bioavailability of rifampicin in FDCs, single drug formulations, or a comparative analysis of the two types of formulation; and (b) studies that compared the efficacy and / or adverse effects of rifampicin in FDCs with rifampicin in single drug formulation. The bioequivalence of rifampicin in FDCs studied was defined as the area under the concentration-time curve (AUC) $(0-24 \mathrm{~h})$ of $80-125 \%$ of the reference product; in most studies, the latter was the loose / single drug rifampicin formulation with the same dose.

As information was obtained from that available in the public domain, no ethical approval was considered necessary for this work.

\section{Results}

More than 450 original peer-reviewed articles, systematic reviews, and reports were assessed for this analysis. Eleven papers from a number of high-TBburden countries, including South Africa, India, and China (Table 1), expressed significant concerns about rifampicin bioavailability, both within FDCs and as a single formulation; these are discussed below in detail. Various authors documented multiple factors associated with low bioavailability, including formulation, storage environment, drug quality, patient age, sex, and concomitant diseases. Most of the studies reviewed from the perspective of efficacy (Table 2) did not find a significant difference in the clinical efficacy or adverse effects of rifampicin in FDCs when compared to single drug formulations.

\section{Rifampicin Bioavailability in Single Formulations versus FDCs}

Table 1 provides a summary of studies which point 
Table 1. Reported Differences in Rifampicin Bioavailability: FDCs versus Single Drug Formulations

\begin{tabular}{|c|c|c|c|c|c|c|}
\hline Country & Year & Sample Size & Study Design & Summary Results & Remarks & Source \\
\hline Italy & $\begin{array}{l}1988 \\
1988 \\
1993\end{array}$ & $\begin{array}{l}10 \text { healthy volun- } \\
\text { teers } \\
13 \text { TB patients } \\
6 \text { human volunteers }\end{array}$ & $\begin{array}{l}\text { Open Crossover } \\
\text { Daily therapy } \\
\text { FDCC }\end{array}$ & $\begin{array}{l}\text { No major PK interactions be- } \\
\text { tween drugs in FDCs or single } \\
\text { drug formulations }\end{array}$ & $\begin{array}{l}\text { Results consistent across the three } \\
\text { types of study populations }\end{array}$ & {$[8-10]$} \\
\hline India & 1999 & $\begin{array}{l}18 \text { healthy volun- } \\
\text { teers }\end{array}$ & $\begin{array}{l}\text { Open cross-over } \\
\text { study }\end{array}$ & $\begin{array}{l}\text { No significant difference in } \\
\text { Rif PK: FDC vs single drug } \\
\text { formulation }\end{array}$ & $\begin{array}{l}\text { Dosage of individual drugs and FDC } \\
\text { not as per body weight }\end{array}$ & [11] \\
\hline India & 2003 & 8 healthy volunteers & $\begin{array}{l}\text { Partially balanced } \\
\text { incomplete block, } \\
\text { random Rif allo- } \\
\text { cation }\end{array}$ & $\begin{array}{l}\text { Reduced BA of Rif in presence } \\
\text { of INH }\end{array}$ & $\begin{array}{l}\text { Excipients in INH combination ruled } \\
\text { out as cause of reduced BA }\end{array}$ & [12] \\
\hline India & 2001 & 6 healthy volunteers & $\begin{array}{l}\text { Single dose, two } \\
\text { treatment, two } \\
\text { period, cross-over }\end{array}$ & $\begin{array}{l}28-32 \% \text { reduced BA of Rif and } \\
\text { analogues in FDC }\end{array}$ & $\begin{array}{l}\text { Possibly due to reduced Rif stability } \\
\text { with INH in gastric environment }\end{array}$ & [13] \\
\hline South Africa & 2018 & $\begin{array}{l}20 \text { healthy volun- } \\
\text { teers }\end{array}$ & $\begin{array}{l}\text { Open-label, three- } \\
\text { way cross-over }\end{array}$ & $\begin{array}{l}20 \% \text { reduced BA in 4-drug } \\
\text { formulation }\end{array}$ & 2-drug formulation bioequivalent & [14] \\
\hline India & 2004 & Review study & $\begin{array}{l}\text { Review of Rif BA in } \\
\text { FDCs }\end{array}$ & $\begin{array}{l}\text { High variability in Rif blood } \\
\text { levels in FDCs }\end{array}$ & $\begin{array}{l}\text { Low Rif BA mainly due to formulation } \\
\text { / substance, rather than variable } \\
\text { absorption }\end{array}$ & [15] \\
\hline South Africa & 2006 & $\begin{array}{l}142 \text { TB patients ( } 138 \\
\text { with known formu- } \\
\text { lation) }\end{array}$ & $\begin{array}{l}\text { TB treatment } \\
\text { cohort }\end{array}$ & $\begin{array}{l}\text { Rif BA mainly affected by } \\
\text { formulation }\end{array}$ & $27 \%$ reduced $B A$ with HIV infection & [16] \\
\hline Mexico & 2010 & $\begin{array}{l}18 \text { healthy volun- } \\
\text { teers }\end{array}$ & $\begin{array}{l}\text { Two-period, } \\
\text { two-sequence } \\
\text { crossover }\end{array}$ & $\begin{array}{l}\text { Rif BA in reference (sin- } \\
\text { gle-drug) formulation 4-fold } \\
\text { higher than FDC }\end{array}$ & $\begin{array}{l}\text { Rif levels below target range, high } \\
\text { variability in FDC }\end{array}$ & [17] \\
\hline China & 2015 & $\begin{array}{l}18+20 \text { healthy } \\
\text { volunteers }\end{array}$ & $\begin{array}{l}\text { Two open-label, } \\
\text { randomized 2-peri- } \\
\text { od crossover / one } \\
\text { 3-period crossover, } \\
\text { respectively }\end{array}$ & $\begin{array}{l}\text { For Rif, only } 1 \text { of } 4 \text { FDC } \\
\text { formulations bioequivalent } \\
\text { to reference, other } 3 \text { had } \\
\text { reduced BA }\end{array}$ & $\begin{array}{l}3 \text { of } 4 \text { FDC products in acceptable } \\
\text { therapeutic range for Rif }\end{array}$ & [18] \\
\hline
\end{tabular}

Notes: FDC: Fixed Dose Combinations; PK: pharmacokinetics; Rif: rifampicin; BA: bioavailability

Table 2. Efficacy of Rifampicin in Fixed Dose Combinations versus single drug formulations

Participant or Population: Patients treated for pulmonary TB

Setting: Hospitals and health centers for TB treatment

Intervention: Fixed-Dose Combinations (FDC)

Comparison: Single-drug formulations

\begin{tabular}{|c|c|c|c|c|c|c|}
\hline Year & Outcome measure & Risk Ratio (RR) & $95 \% \mathrm{Cl}$ & RCTs & $\mathbf{N}$ & Source \\
\hline 2013 & Treatment failure or disease relapse (All) & 1.28 & $0.99-1.7$ & 13 & Unknown & [19] \\
\hline 2013 & Sub group: Patients with baseline drug sensitive tuberculosis & 1.48 & $1.04-2.09$ & 6 & Unknown & [19] \\
\hline 2013 & Sub group: patients receiving self-administered therapy & 1.94 & $1.05-3.57$ & 6 & Unknown & [19] \\
\hline 2016 & Disease relapse & 1.28 & $1.00-1.64$ & 10 & 3621 & {$[20]$} \\
\hline 2016 & Treatment failure & 1.28 & $0.82-2.00$ & 7 & 3606 & {$[20]$} \\
\hline 2016 & Acquired drug resistance & 0.76 & $0.15-3.77$ & 3 & 491 & [20] \\
\hline 2013 & Acquired drug resistance & 1.60 & $0.5-5.4$ & 6 & 2518 & {$[20]$} \\
\hline 2016 & Serious Adverse drug events & 1.45 & $0.90-2.33$ & 6 & 3388 & {$[20]$} \\
\hline 2016 & Adverse events leading to discontinuation of therapy & 0.96 & $0.56-1.66$ & 13 & 5530 & {$[20]$} \\
\hline 2016 & Sputum smear or culture conversion & 0.99 & $0.96-1.02$ & 7 & 2319 & {$[20]$} \\
\hline 2013 & Culture conversion after 2 months of treatment & 1.03 & $1.01-1.04$ & 12 & 4797 & [19] \\
\hline 2016 & Death & 0.96 & $0.67-1.39$ & 11 & 4800 & {$[20]$} \\
\hline
\end{tabular}

Notes: $\mathrm{Cl}$ : Confidence Interval; N: total sample size; RCT: randomized controlled trials

to reduced bioavailability of rifampicin in fixed-dose formulations. Most of the studies conducted from 19802000 did not show any negative effects associated with the combined use of anti-TB drugs in FDCs. Three studies by Acocella et $\mathrm{al}^{8-10}$ compared bioavailability of isoniazid (INH), rifampicin (RIF) and pyrazinamide in free combination or fixed-triple formulations. The number of study subjects varied from 6 to 13. No negative 
pharmacokinetic interactions were found when drugs were administered in fixed combinations. Similarly, in 1999, Gurumurthy et $\mathrm{al}^{11}$ conducted an open cross over study with 18 healthy individuals and concluded that combining anti-tuberculosis drugs into single formulations does not change their pharmacokinetics. In 2003 Immanuel et al. $^{12}$ examined the bioavailability of rifampicin in the presence of isoniazid, ethambutol, pyrazinamide or a combination of the three drugs. This study, which included eight healthy individuals, found that isoniazid alone or in combination with ethambutol and pyrazinamide reduced the bioavailability of rifampicin. This could possibly be explained by degradation of rifampicin in the presence of isoniazid. In a similar study where FDCs were compared with rifampicin alone formulations, nearly $30 \%$ reduction in the bioavailability of rifampicin has been reported ${ }^{13}$. In a recent study by Court et al., the authors performed an open-label, three-way cross-over study of three licensed rifampicin-containing formulations widely used in South Africa to evaluate the bioavailability of rifampicin in a twodrug fixed-dose combination tablet (2FDC) and a four-drug FDC (4FDC) against a single-drug reference. The results of this study indicated an average $20 \%$ reduction in rifampicin bioavailability in the 4FDC, while the $2 \mathrm{FDC} /$ reference was bioequivalent ${ }^{14}$.

A review of eight bioequivalence studies of FDCs containing rifampicin by Agrawal et al in India in 2004 found that FDCs show higher variability in rifampicin blood levels compared to single formulations, which was mainly attributed to complexity in manufacturing FDCs containing rifampicin ${ }^{15}$. Further, this review indicated that low bioavailability of rifampicin in the products studied was mainly due to issues related to drug formulation or drug substance, rather than variability of absorption. Low rifampicin bioavailability in FDCs was found by McIlleron et $\mathrm{al}^{16}$ in a South African study in 2006. In this study, plasma concentration-time profiles of four primary TB drugs (isoniazid, rifampicin, ethambutol, pyrazinamide) were obtained in 142 patients with drug-sensitive TB at two months of daily treatment. Twenty one percent of 138 patients (with known formulation type) were on FDCs while the remaining $79 \%$ were on individual drugs. FDC formulations were found to be associated with a lower bioavailability of rifampicin compared to individual formulations. $\mathrm{AUC}_{0-8}$ (area under the plasma concentrationtime curve in $0-8 \mathrm{~h}$ ) reductions of $8.69 \mathrm{mg} \mathrm{h} / \mathrm{l}(\mathrm{P}=0.001)$ among male patients, $8.37 \mathrm{mg} / \mathrm{l}(\mathrm{P}=0.004)$ among those who received FDC products, and $8.34 \mathrm{mg} \mathrm{h} / \mathrm{l}(\mathrm{P}=$ 0.051) among HIV-infected individuals, respectively, were demonstrated.

In 2010 Milan-Segovia et $\mathrm{al}^{17}$ also found delayed absorption and markedly inferior BA of rifampicin in generic 3-drug FDCs (the test product) approved for use in Mexico compared to a single formulation (the reference product). The number of study subject was limited to 18 healthy individuals. They underwent a randomized twotreatment, two-period crossover double-blind study with a 7-day washout period between treatments. Mean pharmacokinetic parameter values obtained for the test and reference product were $3.13 \pm 2.01 \mu \mathrm{g} / \mathrm{ml}$ and 9.95 $\pm 2.66 \mu \mathrm{g} / \mathrm{ml}$ respectively for peak plasma concentration $\left(\mathrm{C}_{\max }\right), 15.51 \pm 9.77 \mu \mathrm{g} . \mathrm{h} / \mathrm{ml}$ and $58.03 \pm 16.1 \mu \mathrm{g} . \mathrm{h} / \mathrm{ml}$ for area under the concentration time curve (AUC) to the last measurable concentration $\left(\mathrm{AUC}_{0-12 \mathrm{~h}}\right)$ and $17.92 \pm 10.66$ and $68.43 \pm 22.39 \mu \mathrm{g} . \mathrm{h} / \mathrm{ml}$ for AUC up to time infinity $\left(\mathrm{AUC}_{0-\infty}\right)$. The test/reference ratio of the means $(90 \% \mathrm{CI})$ was $25.36 \%$ (17.33-37.10) for $\mathrm{C}_{\max }, 21.25 \%$ (14.61-30.89) for $\mathrm{AUC}_{0-12 \mathrm{~h}}$ and $22.08 \%$ (15.44-31.56) for $\mathrm{AUC}_{0-\infty}$.

A study by Zhu et $\mathrm{al}^{18}$ in China recruited two groups of healthy male volunteers (sample sizes 18 and 20) in an open-label, randomized 2 or 3-period crossover study of four FDCs $(A=4$-drug combination, B, C, D = 2-drug combinations). The relative bioavailability of rifampicin in the FDCs was compared to a separate formulation of rifampicin, the latter used as a reference. The washout period between crossovers was one-week. Mean pharmacokinetic parameter values of rifampicin obtained for formulations A, B, C, and D products were 11.42 \pm 3.41 $\mu \mathrm{g} / \mathrm{ml}, 7.86 \pm 5.78 \mu \mathrm{g} / \mathrm{ml}, 13.05 \pm 6.80 \mu \mathrm{g} / \mathrm{ml}$, and $16.18 \pm$ $3.87 \mu \mathrm{g} / \mathrm{ml}$, respectively, for $\mathrm{C}_{\text {max }}$; and $91.43 \pm 30.82 \mu \mathrm{g} / \mathrm{h} /$ $\mathrm{ml}, 55.49 \pm 37.58 \mu \mathrm{g} / \mathrm{h} / \mathrm{ml}, 96.50 \pm 47.24 \mu \mathrm{g} / \mathrm{h} / \mathrm{ml}$, and $101.47 \pm 33.07 \mu \mathrm{g} / \mathrm{h} / \mathrm{ml}$, respectively, for $\mathrm{AUC}_{0-24 \mathrm{~h}}$. Only formulation A was bioequivalent to the reference product, while the other FDCs were inferior (i.e., they failed the bioequivalence criteria).

\section{Comparative Efficacy of Rifampicin in FDCs versus Single Formulations}

Two recently completed comprehensive systematic reviews and meta-analyses examining FDCs versus separate drugs conducted by Albanna et $\mathrm{al}^{19}$ in 2013 and Gallardo et $\mathrm{al}^{20}$ in 2016 found little or no difference between FDCs and single-drug formulations for most outcomes reported including acquisition of drug resistance, serious adverse events, or adverse effects leading to therapy discontinuation etc. (Table 2). These reviews again support use of FDCs versus single drugs for treatment of pulmonary tuberculosis, but were limited by the lack of data on bioavailability of rifampicin. Despite the high efficacy of FDCs, concerns remain about the risks due to reduced BA of rifampicin in such formulations. Several research studies, surveys and field experiences indicate that the BA of rifampicin may be reduced due to many other factors, potentially including quality of therapeutic substances, drug formulation, storage conditions and meal intake $\mathrm{ki}^{21-26}$. 


\section{Rifampicin Solubility}

Rifampicin drug substance is known to show polymorphism, with polymorphs differing substantially in their rates of dissolution ${ }^{27}$. Given this phenomenon, it is important that the correct crystal form be selected to ensure proper dissolution. Variation in crystal form may cause variation in bioavailability, in formulations with rifampicin alone or in FDCs, between batches from a given manufacturer and from one manufacturer to another, depending on the polymorphic form of the drug product and any polymorphic changes that occur during drug product formulation. The problem may be amplified in the case of FDCs, since the formulations must try to ensure the proper dissolution of all the active substances, resulting in constraints that may limit the ability of the formulations to enhance the rate of dissolution for rifampicin relative to a formulation containing only rifampicin as the active ingredient.

Rifampicin is absorbed well from the stomach, while isoniazid is predominantly absorbed in the intestinal environment ${ }^{28}$. Importantly, rifampicin has shown instability in the presence of dissolved isoniazid in acidic conditions, such as the gastric environment, ultimately impairing rifampicin bioavailability in this combination treatment. It has been reported that rifampicin in the presence of isoniazid undergoes significant decomposition under acidic conditions (existing in the stomach), as compared to rifampicin alone ${ }^{29}$. The decomposition of rifampicin under acidic conditions varies from $8.5 \%$ to $50 \%$ in the time range corresponding to the normal gastric residence time in humans ${ }^{30}$.

\section{FDC Formulation Stability}

A laboratory stability study of four FDC formulations by Singh et $\mathrm{al}^{31}$ in India in 2003 assessed FDC product stability in strip-packaging as compared to blister-packaging at a temperature of $40^{\circ} \mathrm{C}$ and relative humidity of $75 \%$. FDC products in unpacked conditions showed severe (approximately 60\%) decomposition of rifampicin and extensive physical changes. Thus, inadequate packaging technology that substantially affects FDC quality can be a potential reason for poor bioavailability. Nevertheless, exploring the latter was beyond the scope of this study.

\section{Discussion}

Rifampicin continues to play a key role in the treatment of drug-sensitive tuberculosis; reduction in its bioavailability, especially if it occurs due to use in fixed dose combinations, can adversely affect TB treatment in a large number of patients. Drug related factors (e.g. quality of drug substance), production process, packaging, storage and distribution, and environmental conditions can affect the BA of rifampicin. For this reason, quality assurance of the drug product throughout the manufacturing processes including its entire shelf-life is of utmost importance to prevent undesirable clinical consequences resulting from suboptimal quality of medication. The authors acknowledge that this review does not allow for the establishment of the root causes of the low and/or variable rifampicin BA results observed both in the rifampicin-only and FDC drug products. Poor drug substance quality, stability issues, drugdrug interactions, and poor formulation development may all play a role. In cases of poor BA for the rifampicin-only material, it is easier to narrow down the problem to either an inadequate drug level (formulation error or low quality) or an unacceptable formulation (rate of drug release). It is more difficult to establish the root causes of poor BA of rifampicin in FDCs as many possible factors outlined above need to be considered. NTPs need to generate additional evidence to inform policies on FDCs use at national, subnational and individual levels. It is highly recommended that manufacturers combine routine monitoring studies for pharmacovigilance of anti-TB drugs with BA/PK studies for rifampicin and FDCs. Collection of additional data on pharmacokinetics and other information combined with post-marketing surveillance studies is also important for the manufacturers. Results of these studies should be shared in a timely manner across the TB community, nationally and internationally, to inform future policy and programmatic decisions for improved treatment outcome. The authors also acknowledge that the present study is unable to fully assess sources of bias in individual studies that may have led to the observation of low BA of rifampicin in FDCs. However, an attempt was made to minimize bias in the selection of the studies shortlisted for presentation in the paper by having at least two authors review the 451 references that were identified from PubMed, related databases, and international websites including WHO.

The first step to ensuring better FDC quality is being alert to the possibility of lowered BA of rifampicin and other anti-TB drugs. NTPs must continue to insist on procuring only WHO-prequalified drugs, preferably through the Global Drug Facility (GDF); NTPs must also periodically test FDCs for BA / BE (e.g., through clinical studies or clinicallyrelevant dissolution studies) or conduct population-based PK studies of FDCs. Proper storage and distribution of FDCs as per guidelines should be ensured. Finally, the NTP should educate clinical staff and health workers on the importance of maintaining drug quality through monitoring of adverse drug events and treatment outcomes.

Currently, most of the high TB burden countries can access quality assured TB drugs through the GDF procurement mechanism supported by the Global Fund. Despite good economic progress, TB is still prevalent and a significant public health problem in many high-burden countries. The anticipated graduation of these countries 
from the Global Fund poses significant challenges as not all countries are prepared to successfully take over the responsibility for quality assured drug procurement and adequate supply chain management. In such circumstances establishing regional coordination mechanisms for drug quality monitoring can be an effective solution. There is a need to establish Regional Centers of Excellence (COE) to assess quality of FDCs and study rifampicin BA in collaboration with international experts as well as potential involvement of appropriate local contract research organizations (CROs). In terms of logistics, it will be necessary to determine their location and develop suitable international collaborations with expert groups who are conversant with this area. COEs should conduct in vivo bioequivalence or other PK studies in accordance with the guidelines of the WHO Prequalification Team. Developing a clinically relevant dissolution test and making it systematically available to high TB burden countries can be an effective solution to the problem. Good Laboratory Practices (GLP) and Good Clinical Practices (GCP) must be followed in the relevant studies and Good Manufacturing Practices (GMP) must be followed in the preparation of the drug products. The COE network should establish common standard operating procedures (SOPs) to ensure uniform clinical, bioanalytical, pharmacokinetic, and statistical frameworks and reporting. If field studies indicate substandard BA of drugs in specific batches, actions need to be taken. These might include dose adjustments and use of alternative or additional drug products, with review and scientific support from COEs, international agencies (e.g. WHO, IUATLD) and the NTP.

WHO and IUATLD recommend the use of FDCs as an important step to ensure proper treatment of TB. The rationale for use of FDCs is that it simplifies the prescription of drugs, management of drug supply, and may also limit the risk of drug-resistant tuberculosis because of inappropriate drug selection and monotherapy ${ }^{32}$. Recent studies and international documents as discussed above point to a problem with low bioavailability of rifampicin in fixed dose combinations. However, in the field, it remains a "hidden factor" leading to poor treatment outcomes, and the problem often goes unrecognized. It is difficult to study this issue thoroughly unless (a) there is awareness among TB program personnel of its existence; and (b) adequate laboratory and research support is available to NTPs. In stemming the tide of multi-drug resistance (MDR) and extensive drug resistance (XDR), it is paramount to ensure that bioavailability of rifampicin is adequate in any FDC formulation of first-line anti-TB drugs.

Capacity building of health care providers in adequate FDC administration is warranted to ensure timely recognition and elimination of possible causes of low absorption or uptake of a drug within FDCs (e.g., food effect issues), or institute dose adjustment if low uptake has been demonstrated. These areas should be well reflected into the National Training Plans to be implemented by NTPs within the nearest 1 to 3 -year period. National TB programs should also revisit patient education programs and available materials to ensure that patients are aware of and closely follow drug intake and storage instructions.

Close collaboration between NTP and National Drug Authorities (NDA) is a critical precondition for addressing anti TB drugs' quality related concerns. A short to medium term (3-5 years) strategic framework for TB should entail a recommended set of activities to be implemented by NDAs. This could include organizing periodic drug quality/ pharmaceutical audits; ensuring rapid and effective remediation procedures if poor quality material is found; supporting regular BA / PK studies based on WHOrecommended protocol at the central medicine store and regional level combining pharmacovigilance / adverse drug reaction monitoring with BA / PK studies. Countries may decide to seek technical assistance through international agencies for strengthening systems and introducing tools for drug quality assurance as well as revisiting current National TB Strategies to prioritize activities aimed at quality assured treatment for drug-sensitive TB.

\section{Conclusion}

A multitude of recent studies appear to indicate reduced bioavailability of rifampicin either in fixed-dose combinations or in stand-alone preparations of the drug, with the reduction dependent on a variety of factors, which need to be studied further. However, the current BA methodology is expensive for national program. There is a need to come up with a simplified approach for carrying out BA studies as part of post-marketing surveillance. Countries experiencing high levels of rifampicin resistance, should plan new, independent BA assessments for FDCs using simplified protocols.

Capacity building of health care providers in adequate FDC administration is warranted to ensure timely recognition and elimination of possible causes of low absorption or uptake of a drug within FDCs (e.g., food effect issues), or institute dose adjustment if low uptake has been demonstrated. These areas should be well reflected into the National Training Plans to be implemented by NTPs within the nearest 1 to 3 -year period. National TB programs should also revisit patient education programs and available materials to ensure that patients are aware of and closely follow drug intake and storage instructions.

\section{Disclaimer}

The views expressed herein are those of the authors and do not necessarily reflect the views of their affiliated organizations. 


\section{Acknowledgments}

The authors would like to express their gratitude to the entire team at the Center for Innovation and Technology, University Research Co., LLC, Bethesda, Maryland, for their support during the preparation of this manuscript.

\section{References}

1. World Health Organization. Anti-Tuberculosis Drug Resistance in the World; Report No. 4. Vol WHO/HTM/TB/2008.3942008.

2. World Health Organization. Global Tuberculosis Report 2017. Geneva WHO. 2017.

3. Sharma SK, Mohan A. Multidrug-resistant tuberculosis: a menace that threatens to destabilize tuberculosis control. Chest. 2006; 130: 261-272.

4. World Health Organization. Guidelines for treatment of drugsusceptible tuberculosis and patient care (2017 update). Geneva2017.

5. The promise and reality of fixed-dose combinations with rifampicin A joint statement of the International Union Against Tuberculosis and Lung Disease and the Tuberculosis Programme of the World Health Organization. Tuber Lung Dis. 1994; 75: 180-181.

6. World Health Organization. Establishing the bioequivalence of Rifampicin in fixed dose formulations containing Isoniazid with or without Pyrazinamide and/or Ethambutol compared to the single drug reference preparations administered in loose combination : model protocol. Geneva1999.

7. Quality assurance: protocol for assessing the rifampicin bioavailability of combined formulations in healthy volunteers. Int J Tuberc Lung Dis. 1999; 3: S284-S285.

8. Acocella G, Nonis A, Perna G, et al. Comparative bioavailability of isoniazid, rifampin, and pyrazinamide administered in free combination and in a fixed triple formulation designed for daily use in antituberculosis chemotherapy. II. Two-month, daily administration study. Am Rev Respir Dis. 1988; 138: 886-890.

9. Acocella G, Nonis A, Gialdroni-Grassi G, et al. Comparative bioavailability of isoniazid, rifampin, and pyrazinamide administered in free combination and in a fixed triple formulation designed for daily use in antituberculosis chemotherapy. I Single-dose study Am Rev Respir Dis. 1988; 138: 882-885.

10. Acocella G, Luisetti M, Grassi GG, et al. Bioavailability of isoniazid, rifampicin and pyrazinamide (in free combination or fixed-triple formulation) in intermittent antituberculous chemotherapy. Monaldi Arch Chest Dis. 1993; 48: 205-209.

11. Gurumurthy P, Ramachandran G, Vijayalakshmi S, et al. Bioavailability of rifampicin, isoniazid and pyrazinamide in a triple drug formulation: comparison of plasma and urine kinetics. Int J Tuberc Lung Dis. 1999; 3: 119-125.

12. Immanuel C, Gurumurthy $P$, Ramachandran $G$, et al. Bioavailability of rifampicin following concomitant administration of ethambutol or isoniazid or pyrazinamide or a combination of the three drugs. Indian J Med Res. 2003; 118: 109-114.

13. Shishoo CJ, Shah SA, Rathod IS, et al. Impaired bioavailability of rifampicin in presence of isoniazid from fixed dose combination (FDC) formulation. Int J Pharm. 2001; 228: 53-67.

14. Court R, Chirehwa MT, Wiesner L, et al. Quality assurance of rifampicin-containing fixed-drug combinations in South Africa: dosing implications. Int J Tuberc Lung Dis. 2018; 22: 537-543.

15. Agrawal S, Singh I, Kaur KJ, et al. Bioequivalence trials of rifampicin containing formulations: extrinsic and intrinsic factors in the absorption of rifampicin. Pharmacol Res. 2004; 50: 317-327.

16. McIlleron $\mathrm{H}$, Wash $\mathrm{P}$, Burger $\mathrm{A}$, et al. Determinants of rifampin, isoniazid, pyrazinamide, and ethambutol pharmacokinetics in a cohort of tuberculosis patients. Antimicrob Agents Chemother. 2006; 50: $1170-1177$

17. Milan-Segovia RC, Dominguez-Ramirez AM, Jung-Cook H, et al. Relative bioavailability of rifampicin in a three-drug fixed-dose combination formulation. Int J Tuberc Lung Dis. 2010; 14: 1454-1460.

18. Zhu H, Guo SC, Hao LH, et al. Relative bioavailability of rifampicin in four Chinese fixed-dose combinations compared with rifampicin in free combinations. Chin Med J Engl. 2015; 128: 433-437.

19. Albanna AS, Smith BM, Cowan D, et al. Fixed-dose combination antituberculosis therapy: a systematic review and meta-analysis. Eur Respir J. 2013; 42: 721-732.

20. Gallardo CR, Rigau Comas D, Valderrama Rodriguez A, et al. Fixeddose combinations of drugs versus single-drug formulations for treating pulmonary tuberculosis. Cochrane Database Syst Rev. 2016; Cd009913.

21. Blomberg B, Spinaci S, Fourie B, et al. The rationale for recommending fixed-dose combination tablets for treatment of tuberculosis. Bull. World Health Organ. 2001; 79: 61-68.

22. Beckett AH. A general survey of quality control of drugs for tuberculosis. Tubercle. 1968; 49: Suppl88.

23. Hemanth Kumar AK, Kannan T, Chandrasekaran V, et al. Pharmacokinetics of thrice-weekly rifampicin, isoniazid and pyrazinamide in adult tuberculosis patients in India. Int J Tuberc Lung Dis. 2016; 20: 1236-1241.

24. Schipani A, Pertinez H, Mlota R, et al. A simultaneous population pharmacokinetic analysis of rifampicin in Malawian adults and children. Br J Clin Pharmacol. 2016; 81: 679-687.

25. World Health Organization. WHO Expert Committee on specifications for pharmaceutical preparations (Fiftieth report). WHO technical report series; no. 996. Geneva2016.

26. Kumar AKH, Chandrasekaran V, Kumar AK, et al. Food significantly reduces plasma concentrations of first-line anti-tuberculosis drugs. Indian J Med Res. 2017; 145: 530-535.

27. Henwood SQ de Villiers MM, Liebenberg W, et al. Solubility and dissolution properties of generic rifampicin raw materials. Drug Dev Ind Pharm. 2000; 26: 403-408.

28. Mariappan TT, Singh S. Regional gastrointestinal permeability of rifampicin and isoniazid (alone and their combination) in the rat. Int J Tuberc Lung Dis. 2003; 7: 797-803.

29. Shishoo CJ, Shah SA, Rathod IS, et al. Impaired bioavailability of rifampicin in presence of isoniazid from fixed dose combination (FDC) formulation. Int J Pharm. 2001; 228: 53-67.

30. Singh S, Mariappan TT, Sharda N, et al. The Reason for an Increase in Decomposition of Rifampicin in the Presence of Isoniazid under Acid Conditions. Pharmacy and Pharmacology Communications. 2000; 6: 405-410.

31. Singh S, Mohan B. A pilot stability study on four-drug fixed-dose combination anti-tuberculosis products. Int J Tuberc Lung Dis. 2003; 7: 298-303.

32. Bjorn B, Sergio S, Bernard F, et al. The rationale for recommending fixed-dose combination tablets for treatment of tuberculosis. Bulletin of the World Health Organization. 2001; 79 (1). 